\title{
Gasping Syndrome
}

National Cancer Institute

\section{Source}

National Cancer Institute. Gasping Syndrome. NCI Thesaurus. Code C118508.

A syndrome in preterm neonates exposed to benzyl alcohol preservative in intravascular solutions that is characterized by unremitting gasping respirations and may include anion gap metabolic acidosis, neurologic deterioration, renal failure, convulsions, intraventricular hemorrhage, and cardiovascular collapse. 